\title{
The Transitivity AND Asymmetry of Actual Causation
}

\author{
SANDER BECKERS \\ Cornell University \\ JOOST VENNEKENS \\ University of Leuven
}

\begin{abstract}
The counterfactual tradition to defining actual causation has come a long way since Lewis started it off. However there are still important open problems that need to be solved. One of them is the (in)transitivity of causation. Endorsing transitivity was a major source of trouble for the approach taken by Lewis, which is why currently most approaches reject it. But transitivity has never lost its appeal, and there is a large literature devoted to understanding why this is so. Starting from a survey of this work, we will develop a formal analysis of transitivity and the problems it poses for causation. This analysis provides us with a sufficient condition for causation to be transitive, a sufficient condition for dependence to be necessary for causation, and several characterisations of the transitivity of dependence. Finally, we show how this analysis leads naturally to several conditions a definition of causation should satisfy, and use those to suggest a new definition of causation.
\end{abstract}

\section{Introduction}

Causal modelling has become ubiquitous in Artificial Intelligence circles, and is gaining popularity in other fields as well. An unsolved problem in this context is how to define actual causation, i.e., when should we say that one event caused another? Ever since Lewis (1973) first analyzed this problem in terms of counterfactual dependence forty years ago, philosophers and researchers from the Artifical Intelligence community alike have been trying to improve on his attempt at cracking this causal nut. The seminal work of Halpern and Pearl (2005) has led to the structural equations framework becoming the most important language to deal with this problem.

Contact: Sander Beckers <Sander.Beckers@cornell.edu>, Joost Vennekens <Joost.Vennekens@cs.kuleuven.be> 
The currently most prominent approaches to defining actual causation are those within the counterfactual dependence tradition, which started with Lewis (1973). All of these approaches take as their starting point the assumption that counterfactual dependence is sufficient for causation, but not necessary (Hitchcock 2001: Woodward 2003: Hall 2004; 2007; Halpern \& Pearl 2005: Halpern 2016a; Weslake in press: Beckers \& Vennekens in press). That dependence is sufficient is usually accepted simply as a fundamental principle underlying causation. That it is not necessary, on the other hand, is usually defended by pointing to intuitively strong counterexamples. Lewis (1973) forms an important exception to this rule, as he defends the lack of necessity by invoking a principle as well, namely that causation is transitive: causation is transitive, dependence is not, therefore there can be causation without dependence.

The first strategy, that of offering counterexamples, has proven most successful. There are two reasons for this. First, almost everyone besides Lewis rejects the transitivity of causation. Second, there are counterexamples to the necessity of dependence that have nothing to do with transitivity. Despite its success, this strategy has to date not offered a general insight into precisely when or why the transitivity of causation breaks down. Although a substantial number of authors have addressed the problem of transitivity, none of them offers a generally sufficient condition for causation to be transitive (McDermott 1995: Hall 2000; 2004; Hitchcock 2001; Sartorio 2005; Halpern \& Pearl 2005; Halpern 2016b; Paul \& Hall 2013). A recent discussion by Halpern (2016b) does formulate several sufficient and necessary conditions for transitivity, however those apply only to cases where there is dependence.

The main contribution of this paper is to offer a principled explanation of why transitivity should be rejected as a general condition, while also offering conditions under which it should be satisfied. Specifically, we will explain both why the transitivity of causation has a strong appeal, and why there are nevertheless convincing counterexamples to accepting it. We do so by an appeal to the principle that causation is asymmetrical: an event is a cause only if its absence would not have been a cause. ${ }^{1}$ Accepting this principle leads the way to an analysis of causation as a transitive relation compromised by asymmetry. This analysis provides us with a sufficient condition for causation to be transitive, a sufficient condition for dependence to be necessary, and several sufficient and necessary conditions for dependence to be transitive. Finally, we use this analysis to suggest a new definition of causation. The starting point for our analysis consists of a detailed overview of the literature on this topic.

Our present story will be incomplete: we ignore one important type of ex-

1. Note that usually the asymmetry of a relation $R(x, y)$ is interpreted as the following condition: $R(x, y) \Rightarrow \neg R(y, x)$. Here we take it to mean the following instead: $R(x, y) \Rightarrow$ $\neg R(\neg x, y)$. 
ample, Late Preemption, that highlights the temporal aspects of causation. This caveat will not undermine the current discussion, because it stands orthogonal to the issue of transitivity. However, one can integrate this temporal aspect into our analysis, which is what we have done elsewhere (Beckers \& Vennekens in press). There we develop a more refined definition of actual causation, but do not tackle the issue of transitivity. Therefore both papers complement each other. Nevertheless the current paper is entirely self-contained.

The next section introduces the structural equations framework. Section 3 offers the relevant background, and presents a survey of the literature on transitivity. This leads us to suggest a first condition any definition of causation should satisfy, in Section 4 . Section 5 introduces the concept of contributing, which leads the way to a sufficient condition for the transitivity of causation. We discuss the asymmetry of causation in Section 6 and show how it can be combined with transitivity to form an elegant explanation of all aspects here discussed.

\section{Structural Equations Modelling}

We briefly introduce a simple version of structural equations modelling, which is the most popular formal language used to represent causal models. In general, structural equations allow functional dependencies between continuous variables, or discrete variables with possibly an infinite domain. However, the actual causation literature typically considers only examples made up of discrete variables with a finite domain, and propositional formulas. Further, in the majority of cases the variables are Boolean. This is why we restrict attention to those kinds of models. For a detailed introduction, see Pearl (2000).

A structural model consists of a set of endogenous variables $\vec{V}$, a set of exogenous variables $\vec{U}$, and a causal model $M$. Although we only consider models with Boolean variables, we should point out that the results we will present can easily be generalized to allow for multi-valued variables as well. We explain this below.

A model $M$ is a set of structural equations so that there is exactly one equation for each variable $V_{i} \in \vec{V}$. An equation takes the form $V_{i}:=\phi$, where $\phi$ is a propositional formula over $\vec{V} \cup \vec{U}$. For any variable $V_{i}$, we denote by $\phi_{V_{i}}$ the formula in the equation for $V_{i}$ in $M$. We follow the customary practice of leaving the equations for variables that depend directly on the exogenous variables implicit, and simply state the value they take in each particular story.

For an assignment $(\vec{v}, \vec{u})$ of values to the variables in $\vec{V} \cup \vec{U}$, we denote by $\phi^{(\vec{v}, \vec{u})}$ the truth value obtained by filling in the truth values $(\vec{v}, \vec{u})$ in the formula $\phi$. An assignment $(\vec{v}, \vec{u})$ respects $M$, if for each endogenous variable $V_{i}$, its value $v_{i}=$ $\phi_{V_{i}}^{(\vec{v}, \vec{u})}$. As usual, we only consider models $M$ in which the equations are acyclic, which implies that for each assignment $\vec{u}$ to $\vec{U}$, there is exactly one assignment 
$(\vec{v}, \vec{u})$ that respects $M$. Therefore, we refer to $\vec{U}=\vec{u}$ as a context. For every value $\vec{u}$ of $\vec{U}$, we call the pair $(M, \vec{u})$ a causal setting. We write $(M, \vec{u})=\phi$ if $\phi^{(\vec{v}, \vec{u})}=$ true for the unique assignment $(\vec{v}, \vec{u})$ that respects $M$.

A literal $L$ is a formula of the form $V_{i}=v_{i}$ or $U_{i}=u_{i}$. Our restriction to Boolean variables is made concrete here: the only values $v_{i}$ we consider are true and false. Hence our definitions and results can be generalised by simply lifting this restriction. (See the Appendix for some more details.)

We will use the atom $V_{i}$ as a shorthand for $V_{i}=$ true, and the negated atom $\neg V_{i}$ as a shorthand for $V_{i}=$ false. If $V_{i}$ is endogenous, we write $\phi_{L_{i}}$ for $\phi_{V_{i}}$ in both cases.

A causal model $M$ is a tool to represent counterfactual relations between variables, in the sense that changing the values of the variables on the right-side of an equation can change the value of the variable on the left-side, but not vice versa. This makes them suitable devices to model interventions on an actual setting, meaning changes to the value of a variable $V_{i}$ that affect only the values of variables that depend on $V_{i}$, but not those on whom $V_{i}$ itself depends.

Syntactically, we make use of the do()-operator introduced by Pearl (2000) to represent such an intervention. For a model $M$ and an endogenous variable $V_{i}$, we denote by $M_{d o\left(V_{i}\right)}$ and $M_{d o\left(\neg V_{i}\right)}$ the models that are identical to $M$ except that the equations for $V_{i}$ are $V_{i}:=$ true and $V_{i}:=$ false, respectively. Hence for a causal setting $(M, \vec{u})$ such that $(M, \vec{u}) \models C$, the causal setting $\left(M_{d o(\neg C)}, \vec{u}\right)$ corresponds to the counterfactual setting resulting from the intervention on $(M, \vec{u})$ that prevents $C$.

Throughout this paper, we take $C$ and $E$ to be endogenous literals, where $C$ is a candidate cause for the effect $E$.

\section{Literature Survey}

In this paper we consider the following approaches to defining causation: Lewis (1973), Hitchcock (2001), Woodward (2003), Hall (2004: 2007), Halpern and Pearl (2005), Halpern (2016a), Weslake (in press), Beckers and Vennekens (in press). All of them take as their starting point the assumption that counterfactual dependence is sufficient for causation. Informally, given that $E$ and $C$ in fact did occur, $E$ is said to be (counterfactually) dependent on $C$ if $E$ would not have occurred without $C$. The counterfactual is here interpreted in the usual nonbacktracking sense, meaning we assume the non-occurrence of $C$ is the result of an intervention $d o(\neg C)$ on the actual story. For matters of simplicity, most authors only consider deterministic examples, meaning the intervention $d o(\neg C)$ results in precisely one counterfactual story. We comply with this custom for the most part of this paper, but in Section 4.1 we also consider non-deterministic dependence: $E$ is possibly counterfactually dependent on $C$ if possibly $E$ would not 
have occurred without $C$. We here present a formal definition of dependence in the deterministic case.

Definition 1. Given a causal setting $(M, \vec{u})$ such that $(M, \vec{u}) \mid=C \wedge E, E$ is counterfactually dependent on $C$ if $\left(M_{d o(\neg C)}, \vec{u}\right) \mid \neg$ E.

We take the sufficiency of dependence as our first principle.

Principle 1 (Dependence). If $E$ is dependent on $C$ in a causal setting $(M, \vec{u})$, then $C$ is a cause of $E$ w.r.t. $(M, \vec{u})$.

There are three basic types of examples which are commonly used to defend the claim that dependence is not necessary, namely Early Preemption, Late Preemption, and Symmetric Overdetermination. Of course there exist many more counterexamples to the necessity of dependence, but in essence they can all be reduced to these paradigmatic cases, or combinations thereof. To illustrate, we present a case of Early Preemption from Hitchcock (2001. 276):

Example 1 (Backup). An assassin-in-traininig is on his first mission. Trainee is an excellent shot: if he shoots his gun, the bullet will fell Victim. Supervisor is also present, in case Trainee has a last minute loss of nerve (a common affliction among student assassins) and fails to pull the trigger. If Trainee does not shoot, Supervisor will shoot Victim herself. In fact, Trainee performs admirably, firing his gun and killing Victim.

The following is the standard model used in the literature for this story, where the context is such that Trainee is true.

$$
\begin{aligned}
\text { Victim } & :=\text { Trainee } \vee \text { Supervisor. } \\
\text { Supervisor } & :=\neg \text { Trainee. }
\end{aligned}
$$

Intuitively it is clear that Trainee is a cause of Victim, yet using this model we see that Victim is not dependent on Trainee. The starting point for any definition of causation in the counterfactual tradition is to provide a way of handling cases of Early Preemption. Lewis (1973) does so by invoking another appealing principle of causation: that it is a transitive relation.

Principle 2 (Transitivity). If $C$ is a cause of $D$ and $D$ is a cause of $E$ w.r.t. $(M, \vec{u})$, then $C$ is a cause of $E$ w.r.t. $(M, \vec{u})$.

Lewis (1973) takes Dependence and Transitivity at face value, and defines causation as the transitive closure of dependence. This definition is able to handle cases of Early Preemption by focussing on an intermediate event in between Trainee's shot and Victim getting hit, for example the event of Trainee's bullet flying through the air. By adding a variable to the model representing this event, 
say Bullet, Lewis gets the desired result: Victim is dependent on Bullet, Bullet is dependent on Trainee, and thus by Transitivity, Trainee causes Victim.

Elegant as it may be, McDermott (1995) demonstrated that there are two major problems with this definition: it is neither a necessary condition for causation, nor a sufficient one. For the former, cases of Late Preemption and Symmetric Overdetermination do not contain a chain of dependencies, and still intuitively exhibit causation. For the latter, there are intuitively convincing counterexamples to the transitivity of causation. The first problem is generally taken to be a decisive blow to Lewis's definition. The second problem, however, has more general repercussions: giving up Transitivity is not taken lightly. To understand why this is the case, we give an overview of the literature on this problem.

\subsection{Counterexamples to Transitivity}

Many authors have tackled the issue of transitivity, and their analysis always has the following two features: the transitivity of causation sounds intuitively appealing, but unfortunately there are convincing counterexamples (McDermott 1995 : Hall 2000; 2004; Hitchcock 2001; Sartorio 2005; Halpern \& Pearl 2005; Halpern 2016b; Paul \& Hall 2013). Halpern is the most recent to take up this view, summarising the importance of transitivity in the counterfactual tradition as follows:

Paul and Hall [2013, p. 215] suggest that "preserving transitivity is a basic desideratum for an adequate analysis of causation". Hall [2000] is even more insistent, saying "That causation is, necessarily, a transitive relation on events seems to many a bedrock datum, one of the few indisputable a priori insights we have into the workings of the concept." Lewis [1986, 200o] imposes transitivity in his influential definition of causality, by taking causality to be the transitive closure ("ancestral", in his terminology) of a one-step causal dependence relation. (Halpern 2016b: 2)

Although Halpern (2016b) agrees that transitivity should be preserved as much as possible, he acknowledges that there are convincing counterexamples, as do all of the other authors mentioned. To illustrate, we present a few of them here.

The first is by Hitchcock (2001), but Hall (2000) gives an almost identical example.

Example 2 (Boulder). A boulder is dislodged, and begins rolling ominously toward Hiker. Before it reaches him, Hiker sees the boulder and ducks. The boulder sails harmlessly over his head with nary a centimeter to spare. Hiker survives his ordeal.

The following is an appropriate model for this story, where the context is 
such that Boulder is true.

$$
\begin{aligned}
\text { Dies }:=\text { Boulder } \wedge \neg \text { Duck. } \\
\text { Duck }:=\text { Boulder. }
\end{aligned}
$$

We see that Hiker surviving ( $\neg$ Dies) is dependent on Duck, and Duck in turn is dependent on Boulder. Hence by Dependence, Boulder causes Duck and Duck causes $\neg$ Dies. However it would be absurd to conclude from this that the boulder coming down is a cause of hiker's survival. Thus this example presents a violation of Transitivity.

The next example is originally due to McDermott (1995: 531), but is also discussed by others (Hall 2000; Hitchcock 2001; Halpern 2016b).

Example 3 (Dog Bite). Terrorist, who is right-handed, must push a detonator button at noon to set off a bomb. Shortly before noon, he is bitten by a dog on his right hand. Unable to use his right hand, he pushes the detonator with his left hand at noon. The bomb duly explodes.

We model this as follows, where the context is such that Bite is true.

$$
\begin{aligned}
\text { Bomb } & :=L H \vee R H . \\
L H & :=\text { Bite. } \\
R H & :=\neg \text { Bite. }
\end{aligned}
$$

Just as with Boulder, it would be absurd to consider the dog bite to be a cause of the explosion, as implied by Dependence and Transitivity.

Next an example from Hall (2000) that is structurally identical to the previous one, and is also discussed by Halpern and Pearl (2005).

Example 4 (Switch). An engineer is standing by a switch in the railroad tracks. A train approaches in the distance. She flips the switch, so that the train travels down the left-hand track, instead of the right. Since the tracks reconverge up ahead, the train arrives at its destination all the same.

The following is an appropriate model for this story, where RT (LT) means that the train goes down the right-hand (left-hand) track, Dest means that the train arrives at its destination, and the context is such that Switch holds, i.e., the engineer flips the switch.

$$
\begin{aligned}
\text { Dest } & :=L T \vee R T . \\
L T & :=\text { Switch. } \\
R T & :=\neg \text { Switch. }
\end{aligned}
$$


Intuitively, flipping the switch is not a cause of the train's arrival, again going against the combined claims of Dependence and Transitivity.

Many more counterexamples are given in the literature, but their structures are very similar to the examples here presented. Given the existence of these intuitively convincing counterexamples, all of the authors mentioned agree that Transitivity should be abandoned. ${ }^{2}$

Although this means we are sacrificing an intuitive property of causation, we should be careful not to sacrifice too much: even if some cases provide convincing counterexamples to transitivity, there is no reason to abandon it altogether. Again we take our cue from Halpern:

In light of the examples, should we just give up on these intuitions? Paul and Hall [2013] suggest that "What's needed is a more developed story, according to which the inference from " $C$ causes $D$ " and " $D$ causes $E$ " to " $C$ causes $E$ " is safe provided such-and-such conditions obtain - where these conditions can typically be assumed to obtain, except perhaps in odd cases." The goal of this paper is to provide sufficient conditions for causality to be transitive. (Halpern 2016b; 2)

Halpern (2016b) only discusses such conditions in case of dependence. By contrast, we provide several necessary and sufficient conditions for dependence to be transitive, and derive from this a sufficient condition for causation to be transitive in general.

\section{The (In)transitivity of Dependence}

By Dependence we know already that whenever dependence is transitive, causation will be transitive as well. In all of the papers mentioned, it holds for all of the counterexamples there discussed, that they have one essential thing in common: they are also counterexamples to the transitivity of dependence. ${ }^{3}$ This leads to the suggestion that likewise, whenever dependence violates transitivity, so does causation. Taken together this amounts to the following Condition:

Condition 1. If $E$ depends on $D$ and $D$ depends on $C$ w.r.t. $(M, \vec{u})$, then it holds that: $C$ causes $E$ w.r.t. $(M, \vec{u})$ iff $E$ depends on $C$ w.r.t. $(M, \vec{u})$.

Any definition which satisfies this condition has the desirable property that it violates transitivity in all of the counterexamples discussed in the literature,

2. Originally Hall did try to hold on to Transitivity, by sacrificing Dependence. Later, he rejected this view Hall 2000: 2007).

3. This is in line with Hitchcock (2001. 276), who defines ordinary cases of causation as those where the transitivity of dependence is respected. 
while also respecting transitivity in ordinary cases where dependence does so as well.

Recall that dependence is not necessary for causation in general, due to problem cases exhibiting Early Preemption, Late Preemption, or Symmetric Overdetermination. The above condition states that in case we have a chain of dependencies, dependence does become a necessary condition. To bring these two observations in agreement requires showing that those problem cases do not occur in case there is a chain of dependencies from $C$ to $D$ to $E$, but no dependence of $E$ on $C$.

Regarding Late Preemption and Symmetric Overdetermination, we shall be brief: there is no example in the literature we know of that is considered a case of either of those, and for which Condition 1 is violated. Moreover, Late Preemption differs from Symmetric Overdetermination only with regards to its temporal properties, which as mentioned earlier are of no relevance to this paper. Therefore we use the following classic example of Symmetric Overdetermination to illustrate our point:

Example 5 (Symmetric Overdetermination). Suzy and Billy both throw a rock at a bottle. Both rocks hit the bottle simultaneously, upon which it shatters. Either rock by itself would have sufficed to shatter the bottle.

We can model this story using the single equation $B S:=$ Suzy $\vee$ Billy, where $B S$ represents the shattering of the bottle, and Suzy (Billy) represents Suzy (Billy) throwing a rock. The context is such that both Suzy and Billy are true. Intuitively both Suzy and Billy are causes of BS, yet BS is not dependent on either of them. It is clear that in this example the failure of dependence has nothing to do with issues of transitivity. Rather, the problem is that there are two completely independent processes which suffice to bring about $E$, and both of them actually occur, overdetermining $E$. Adding more detail by inserting variables in between Suzy and BS, so that there is a chain of causes leading from Suzy to BS, does nothing to change the fact that there will never be a chain of dependencies from Suzy to BS.

\subsection{Early Preemption}

Cases of Early Preemption provide the other reason why dependence is not necessary for causation. Therefore we need to show that such cases do not occur when there is a chain of dependencies, and no dependence of the end of the chain on its start, so that Condition 1 can be accepted.

There is one basic causal setting that is considered by most to be the prototypical case of Early Preemption: it is the setting used for Backup introduced in Section 3 . The model was the following, where the context is such that Trainee 
holds:

$$
\begin{aligned}
\text { Victim } & :=\text { Trainee } \vee \text { Supervisor. } \\
\text { Supervisor } & :=\neg \text { Trainee. }
\end{aligned}
$$

Since there is no chain of dependencies between Trainee and Victim, Condition 1 does not apply and there is no problem with judging Trainee a cause of Victim. We will call this the small model.

Observe however that this model is quite similar to that used for Dog Bite and Switch, two of the counterexamples to the transitivity of causation we discussed earlier. The only difference lies in there being an intermediate variable in between the candidate cause and the effect. In fact, as we mentioned in Section 3 , Lewis's account exploits the similarity between these two models to deal with Early Preemption: he creates a chain of dependencies from Trainee to Victim by adding an intermediate variable Bullet that represents the bullet flying through midair. Doing so results in what we will refer to as the large model:

$$
\begin{aligned}
\text { Victim }: & =\text { Bullet } \vee \text { Supervisor. } \\
\text { Bullet }: & =\text { Trainee. } \\
\text { Supervisor }: & =\neg \text { Trainee. }
\end{aligned}
$$

This model is identical to the ones that we have used for Dog Bite and Switch. Since Condition 1 was formulated precisely to avoid the conclusion that Bite and Switch are causes, applying it to this model likewise leads to the result that Trainee is not a cause of Victim.

We are thus faced with the following problem: the large model is suggested as a model both for examples labelled Early Preemption, in which there is no causation, and for examples labelled Switch, in which there is causation. (By calling an example a Switch, we simply mean that it is an example where intuitively there is no causation, as opposed to Early Preemption.) Therefore the only way out is to argue that the large model is not appropriate for one of these examples.

We present three strategies for arguing that the large model is not appropriate for Early Preemption, and one strategy which argues against it being appropriate for Switch. Two of the first three strategies present an analysis of Early Preemption that does not conflict with Condition 1 . The two remaining strategies do conflict with Condition 1 , but we will argue that they are problematic, and should therefore be avoided.

First, we start with the strategy defended by Weslake (in press), which is the simplest. According to him, the distinguishing feature of Early Preemption is that there is no intermediate variable between the candidate cause and the effect. Therefore only the small model is appropriate for Early Preemption, and only the large model is appropriate for Switch, case closed. 
Second, we discuss our own preferred strategy to handle this problem. (See also Beckers \& Vennekens in press.) We argue against using the large model for Early Preemption, but the argument applies just as well to the small model: we claim that an appropriate model for Early Preemption ought to be non-deterministic. Hence contrary to the first strategy, the presence of an intermediate variable between Trainee and Victim is irrelevant to our strategy. ${ }^{4}$

Specifically, we claim that the underlying motivation behind calling Trainee a cause in the Backup example is that contrary to the two models above, intuitively we do consider it possible that Victim's death is dependent on Trainee's action after all. For instance, it is natural to assume that even Supervisors are not always accurate, or may also have a loss of nerve. Adding these assumptions to the small model gives the following, more appropriate, non-deterministic model:

$$
\begin{aligned}
\text { Victim }: & =\text { Trainee } \vee(\text { Supervisor } \wedge \text { Accurate }) . \\
\text { Supervisor }: & =\neg \text { Trainee } \wedge \neg \text { Nerves. }
\end{aligned}
$$

Here we have added variables to represent the accuracy of Supervisor's shot, and the possibility that he has a loss of nerve. The actual story only gives us the partial context such that Trainee holds, leaving unspecified the values of Accurate and Nerves. Using this model results in there being possible counterfactual stories so that Victim does not die. This implies that possibly Victim is dependent on Trainee, and therefore possibly Trainee is a cause of Victim.

We can make this formally precise by extending the context $\vec{U}$ with exogenous variables $\vec{W}$ (such as Accurate and Nerves) whose values are undetermined in the actual story.

Definition 2. Given a causal model $M$ over endogenous variables $\vec{V}$ and exogenous variables $\vec{U}$, we define a partial context as an assignment $\overrightarrow{u^{\prime}}$ of values to variables so that $\vec{U}^{\prime} \subseteq \vec{U}$, and refer to $\left(M, \vec{u}^{\prime}\right)$ as a partial causal setting. We call an assignment $\vec{w}$ to the remaining exogenous variables $W=U \backslash U^{\prime}$ a completion of $u^{\prime}$.

Further, we will say that something is certain in a partial causal setting $\left(M, \overrightarrow{u^{\prime}}\right)$, if it holds in all causal settings $\left(M, \overrightarrow{u^{\prime}} \cup \vec{w}\right)$ that complete $u^{\prime}$. Likewise, something is possible if it holds in at least one causal setting $\left(M, \overrightarrow{u^{\prime}} \cup \vec{w}\right)$ that completes $u^{\prime}$.

Since Victim is dependent on Trainee in case either $\neg$ Accurate or Nerves holds, by Dependence we get that Trainee is possibly an actual cause of Victim.

One might object that this conclusion is too weak, on the grounds that our intuitive judgment is best understood as the claim that Trainee is certainly an actual cause of Victim. If that is true, then we bite the bullet and accept the fact that our intuitive judgment is wrong. There is, however, a more subtle way to

4. Menzies (2004) also argues that the intermediate variable does not matter, and likewise claims that Trainee is not a cause in either of the two deterministic models. 
understand our intuitive causal judgments that allows them to fall short of certainty, by distinguishing between different levels of information about the actual story. If we have full information, then we are dealing with a complete causal setting, and the notions of certainty and possibility collapse into the actual. In the absence of any information regarding the actual story, on the other hand, we are left with just the causal model to determine the possible actual causes. Therefore the notions of "possible actual cause" and "actual cause" come closer together as a partial context comes closer to a complete context. The formal details that determine when these two notions are close enough to be used interchangeably are to be found in the Appendix, but the idea is, roughly, that we only allow uncertainty regarding exogenous variables whose influence is limited entirely to counterfactual stories. This condition is fulfilled in Early Preemption cases like Backup because the values of Accurate and Nerves only come into play when considering the counterfactual that Trainee would not have shot.

Another possible objection to our strategy is that it amounts to begging the question, since the elements of uncertainty that we have introduced are not part of the original story. Therefore, the objection goes, our solution no longer works if we explicitly stipulate that Victim will die in all of the possible counterfactual stories. Our answer is simple: we agree that in such a case Trainee would indeed not be a cause of Victim.

We believe the problem here lies not with our causal judgments, but with the discrepancy between stipulating that a causal model is deterministic, and our intuition that it is not. By stipulating that certain extremely counterintuitive characteristics hold for a scenario that is greatly underdetermined by its short description, we end up with an example that is too far removed from commonsense for our intuitions to offer any guidance. Concretely, in order to get our intuitions on board with the assumption that the Backup example is truly deterministic requires more than stating that it is impossible for Supervisor to have a loss of nerves, or to miss when he shoots. For starters, we need to imagine a situation where the only possible options for Trainee are to either shoot accurately at Victim, or not to shoot at all. We are to imagine that he is unable to shoot and miss on purpose, or that he shoots Supervisor instead of Victim, or shouts to Victim to seek cover, etc. If we are to rely on our intuitions here at all, we better come up with a more realistic scenario to describe Trainee's predicament. In other words, we need an example where Trainee is faced with a binary choice such that regardless of the choice he makes Victim will die as a result. We don't have to look very far, the following example fits those criteria:

Example 6 (Trainee's Switch). Trainee is standing by a switch in the railroad tracks. A train approaches in the distance. She flips the switch, so that the train travels down the left-hand track, instead of the right. Since the tracks reconverge up ahead, the train arrives at its destination all the same. Unfortunately for Victim, he is tied to the tracks 
at the destination, and is killed by the train.

As was the case for Switch, here it is entirely plausible to assume that the backup process will certainly function properly, i.e., the right track will not break down all of a sudden. Hence here we certainly have an example for which our intuitions agree that Victim's death is inevitable, as opposed to the Backup example. As with Switch, intuitively Trainee flipping the switch is not a cause of Victim's death, which confirms our analysis.

Cases of Early Preemption are distinguished from Switches on a one-by-one basis: is the scenario such that the relevant counterfactual story, in which we have both $\neg C$ and $\neg E$, should be allowed by the model? I.e., is there any reason to doubt that the backup process will function properly? If yes, then possibly there is dependence and we consider it a case of Early Preemption. If no, then the backup process-Supervisor shooting, the functioning of the right hand track, Terrorist using his right hand-is taken to be reliable and it is a Switch. Obviously this distinction involves a certain amount of subjectivity, but given the divergence of intuitions between people regarding the same story we take this to be a benefit of our approach.

Third we consider the strategy by Hall (2007), which is similar to ours, but not quite the same. He also uses the large model only for Switch, whereas his model for Early Preemption contains an extra variable that serves to turn the backup process on or off. As with our strategy, he agrees that the distinction between the two cases comes down to whether or not the backup process can fail. The difference is that on his view of Early Preemption, even if we somehow have evidence that in the actual story the backup process was reliable and would not have failed, we may still consider the counterfactual story in which it does fail. But on this view it becomes quite hard-if not impossible-to express the difference between Early Preemption and a Switch. For every backup process there is some relevant property on which its reliability depends: Supervisor being accurate or not losing his nerves, Terrorist's ability to use his right hand, the right hand track not being broken, etc. All it takes on his account to change a Switch into a case of Early Preemption is to add a variable representing this property. Then, even if we have evidence that the relevant property is present, we may still consider the counterfactual story in which it is not. ${ }^{5}$ Because of this undesirable consequence, we do not find this strategy convincing.

Fourth there is the strategy offered by Hitchcock (2001) and Halpern and Pearl (2005): they argue that both the small and the large model are appropriate for Early Preemption, but neither is appropriate to model switching stories such as Dog Bite and Switch. They are forced to take up this position because their solution to get Trainee to come out as a cause in the small model applies just as

5. Hitchcock 2009. 398) offers a similar criticism. 
well to the large model. In response to the common practice of using the large model for Switches, they argue case by case why on closer inspection that model is not appropriate for a particular story, or why that story should be considered a case of Early Preemption rather than a Switch. Let us examine both replies.

Hitchcock (2001) argues against using the large model for Dog Bite. However, that argument only applies to accounts that make use of so-called "ENF counterfactuals", which are a particular form of interventions on a structural model that we will not go into. ${ }^{6}$ Halpern and Hitchcock (2010: 16) argue against using the large model for Switch, on the basis that the variables $L T$ and $R T$ are logically related: "the train cannot be on both tracks at once". First of all, we disagree that the relation between these variables is logical: it is a matter of physics, not logic, that a train can only occupy a single track at any given moment. Second of all, this argument does not apply to Dog Bite, as one can push a detonator using two hands. In light of this, and in absence of a general argument as to why such models should never be used to model switches, this reply is not convincing.

Regarding the same Switch example, Halpern and Pearl (2005) claim that the large model can be appropriate, but only if we consider the possibility that the right hand track will fail as relevant.

It is this possibility [that of a malfunctioning track] that should enter our mind whenever we decide to designate each track as a separate mechanism (i.e., equation) in the model and, keeping this contingency in mind, it should not be too odd to name the switch position a cause of the train arrival (or non-arrival). (Halpern \& Pearl 2005, 27)

Pearl (2000) makes the same claim regarding a Switch made up of two lamps. The motivation behind this strategy and ours is the same: if we take the failure of the backup process to be a relevant possibility, then we should consider the counterfactual story in which it occurs. The difference is that we do not seek recourse in structural contingencies (such as ENF counterfactuals) to represent such counterfactual stories, but use a partial context to allow for non-deterministic models. It is important to point out that this agreement is limited to the distinction between Switches and Early Preemption, and should not be generalised. Halpern and Pearl (2005) use structural contingencies to consider vastly different counterfactual stories as well, which have nothing to do with the issue at hand.

We have now discussed four of the most important strategies to handling Early Preemption, and presented a number of arguments against adopting the third or fourth strategy. What matters for the subject of this paper, however, is that the first and second strategies are both viable options for handling Early Preemption properly without running into conflict with Condition 1 .

6. See Paul and Hall 2013 Chapter 5) for a detailed discussion of the problems these ENF counterfactuals pose to dealing properly with the counterexamples to Transitivity. 
To sum up, because of the fact that the counterexamples to Transitivity are without exception also counterexamples to the transitivity of dependence, and in light of the lack of opposition from problem cases like Overdetermination and Early Preemption, we claim that Condition 1 should be accepted.

\section{Transitivity in General}

\subsection{Contributing}

A proper understanding of the intransitivity of causation requires looking further than dependence. Dependence stands at one end of a spectrum as a strong but intransitive relation that is sufficient for causation. At the other end there is the concept of contributing, which is a weak and transitive relation. We introduce some new concepts in order to define it, and present it as a necessary condition for causation.

Definition 3. We define that a consistent set of literals $\vec{L}$ is sufficient for a literal $L_{i}$ w.r.t. $M$ if $\wedge \vec{L} \Rightarrow \phi_{L_{i}}$ and $L_{i}$ is positive, or $\wedge \vec{L} \Rightarrow \neg \phi_{L_{i}}$ and $L_{i}$ is negative. Here, $\wedge \vec{L}$ denotes the conjunction of all elements of $\vec{L}$.

For example, in our rock-throwing model for Symmetric Overdetermination, $\{S u z y\}$ is sufficient for $B S$ because $S u z y \Rightarrow S u z y \vee B i l l y$ is a logically valid implication, similarly $\{\neg$ Suzy, $\neg$ Billy $\}$ is sufficient for $\neg B S$ because $\neg$ Suzy $\wedge \neg$ Billy $\Rightarrow$ $\neg($ Suzy $\vee$ Billy $)$ is trivially valid.

A sufficient set as a whole clearly contributes to a literal being true, but its necessary elements are doing all the work.

Definition 4. Given $(M, \vec{u})=C \wedge E$, we define that $C$ is a direct actual contributing cause of $E$ if there exists a set of literals $\vec{L}$ containing $C$, such that $(M, \vec{u})=\vec{L}$ and $\vec{L}$ is sufficient for $E$, but $\vec{L} \backslash\{C\}$ is not. We call $\vec{L}$ a witness for $C$ w.r.t. E.

Note that only literals which appear in the equation for $E$ can ever be direct actual contributing causes. To illustrate, both Suzy and Billy are direct actual contributing causes of BS in Symmetric Overdetermination, with witnesses $\{S u z y\}$ and $\{$ Billy\} respectively. More generally, the connection between two literals need not be direct:

Definition 5. Given $(M, \vec{u}) \models C \wedge E$, we define that $C$ is an actual contributing cause of $E$ if there exist literals $C=L_{1}, \ldots, L_{n}=E$ so that each $L_{i}$ is a direct actual contributing cause of $L_{i+1}$.

From now on we speak simply of $C$ contributing to $E$, rather than saying that $C$ is an actual contributing cause of $E$. Informally, if $C$ does not contribute to $E$, it plays no role in determining the value of $E$. Indeed, we leave it to the reader 
to verify that all the definitions under consideration-mentioned in Section 3 satisfy the following principle:

Principle 3 (Contributing). If $C$ is a cause of $E$ in a causal setting $(M, \vec{u})$, then $C$ contributes to E w.r.t. $(M, \vec{u})$.

Informally, what this principle states is that all actual causes of $E$ are literals that contributed to satisfying/falsifying an equation, which in turn contributed to satisfying/falsifying another equation, etc., which in the end contributed to satisfying/falsifying the equation for $E$.

The following is an interesting connection between dependence and contributing, that will prove useful for interpreting subsequent results.

Theorem 1. E depends on $C$ w.r.t. $(M, \vec{u})$ iff $C$ contributes to $E$ w.r.t. $(M, \vec{u})$ and $\neg C$ contributes to $\neg E$ w.r.t. $\left(M_{d o(\neg C)}, \vec{u}\right)$.

Proofs of all Theorems can be found in the Appendix.

\subsection{A Sufficient Condition for Transitivity}

Condition 1 states that causation and dependence are equally transitive in case we have a chain of dependencies.

The next step is to look at transitivity in case there is a chain of causes simpliciter, but not necessarily a chain of dependencies. More specifically, we want to find a good sufficient condition for the transitivity of causation in general. Since Condition 1 suffices to respect all counterexamples to Transitivity from the literature, a naive suggestion would be to simply demand that causation is always transitive when there is no chain of dependencies. To understand why this would not work, we show how the counterexamples can easily be modified so that there no longer is a chain of dependencies, yet intuition would still find that causation is intransitive. All we need to do is add a little Symmetric Overdetermination into the mix.

Example 7 (Dog Bite with Backup). Imagine the story of the Terrorist from Dog Bite, but with a little twist: there are two detonators that can be pushed, either of which will set off the bomb. To make sure nothing goes wrong, Backup pushes the other detonator at the same moment as Terrorist pushes his.

We can re-use our old model, except that we add Backup's action.

$$
\begin{aligned}
\text { Bomb } & :=L H \vee R H \vee \text { Backup } \\
L H & :=\text { Bite. } \\
R H & :=\neg \text { Bite. }
\end{aligned}
$$


Just as in the original example, Dependence implies that Bite is a cause of $L H$. However, Bomb is now no longer dependent on $L H$, so there is no chain of dependencies from Bite to Bomb and Condition 1 does not apply. Because Bomb is symmetrically overdetermined by both $L H$ and Backup, we also have that $L H$ should still be a cause of Bomb. Nevertheless Bite should still not be considered a cause of Bomb, violating Transitivity.

The lesson learned is that the focus should not be on the presence of a chain of dependencies as such, but rather on the conditions that decide whether or not dependence is transitive. Therefore we now present three different characterisations of the transitivity of dependence.

Theorem 2. If $E$ depends on $D$ and $D$ depends on $C$ w.r.t. $(M, \vec{u})$, then the following statements are all equivalent:

1. E depends on $C$ w.r.t. $(M, \vec{u})$.

2. $\neg$ E depends on $\neg C$ w.r.t. $\left(M_{d o(\neg C)}, \vec{u}\right)$.

3. $\neg C$ contributes to $\neg E$ w.r.t. $\left(M_{d o(\neg C)}, \vec{u}\right)$.

4. $\neg C$ does not contribute to $E$ w.r.t. $\left(M_{d o(\neg C)}, \vec{u}\right)$.

Note that in general, i.e., without the restriction to chains of dependencies, the statements in this theorem are not all equivalent. Rather we have that $1 \Leftrightarrow 2$, and $2 \Rightarrow 3 \Rightarrow 4$, but also $4 \nRightarrow 3 \nRightarrow 2$.

This theorem shows that to satisfy Condition 1 , it suffices to take any of the three last conditions as a sufficient condition for the transitivity of causation. Since Transitivity is intuitively appealing, we want to restrict Transitivity as little as possible. Given that the last condition from Theorem 2 is clearly weaker than the other three (in general), this naturally leads to the following condition:

Condition 2 (Sufficient Condition for Transitivity). If $C$ causes $D$ and $D$ causes $E$ w.r.t. $(M, \vec{u})$, then the following holds:

If $\neg C$ does not contribute to $E$ w.r.t. $\left(M_{d o(\neg C)}, \vec{u}\right)$ then $C$ causes $E$ w.r.t. $(M, \vec{u})$.

In light of Theorem 2 and Dependence, we can interpret Condition 2 informally as stating that a definition of causation ought to be "at least as transitive as dependence", i.e., its sufficiency condition for transitivity should be at least as weak as that for dependence. (Note that this statement can be endorsed without having to accept Condition 国) Before we follow through on this lead, we present an example to show that violations of Condition 2 lead to counterintuitive results. 


\subsection{Counterexample}

To illustrate what goes wrong if Condition 2 is not accepted, we look at two very similar examples using the definitions from Halpern and Pearl (2005), Woodward (2003), and Weslake (in press), none of which satisfies Condition 2.

Example 8 (Assassin). Assassin adds Cyanide to Victim's coffee, which is certain to kill a person. Backup adds a Liquid to the coffee that reacts with Coffee to form Arsenic, another lethal substance. Victim drinks his coffee, which now contains lethal doses of both Cyanide and Arsenic, and dies.

We can model this as follows, where the context is such that Liquid and Cyanide are true:

$$
\begin{aligned}
\text { Dies }: & =\text { Arsenic } \vee \text { Cyanide } . \\
\text { Arsenic } & :=\text { Liquid } \wedge \text { Coffee. }
\end{aligned}
$$

All of the definitions listed in Section 3 -including the three mentioned above-agree that Liquid is a cause of Dies. ${ }^{7}$

By Dependence, Liquid is a cause of Arsenic. Further, as was the case with Symmetric Overdetermination, Arsenic is a cause of Dies. Given that $\neg$ Liquid cannot possibly contribute to Dies, Condition 2 implies that Liquid is a cause of Dies, in agreement with the above definitions.

However, if we change the example only slightly, we get a different result. Imagine that instead of the Liquid reacting with Coffee to form Arsenic, it's the reaction between Liquid and Cyanide that forms Arsenic. In other words, we assume the following model:

$$
\begin{aligned}
\text { Dies } & :=\text { Arsenic } \vee \text { Cyanide } . \\
\text { Arsenic } & :=\text { Liquid } \wedge \text { Cyanide. }
\end{aligned}
$$

For this example the three definitions mentioned do violate Condition 2 , because they no longer judge Liquid to be a cause of Dies. It is hard to see what could possibly justify this change in causal judgment: in both cases Liquid is added to a coffee containing Cyanide, in both cases Liquid reacts with part of that mixture, and in both cases this results in two lethal substances overdetermining Victim's death. Therefore the intuitive result would be that Liquid remains a cause in this example as well, which is guaranteed by accepting Condition 2

7. Strictly speaking the latest definition defended by Halpern (2016a) is an exception, since it judges Liquid to be part of a cause rather than a cause proper. However, he also suggests that these terms ought to be used synonymously. 


\section{Transitivity and Asymmetry}

\subsection{Asymmetry}

Looking back at the model which we identified as a Switch, i.e., the second model we considered in Section 4.1. we note that there is a remarkable symmetry between the actual story and the counterfactual story that we get when intervening on $C$ : in both cases there is a chain of counterfactual dependence from the candidate cause ( $C$ and $\neg C$, respectively) to the effect $E$.

This offers an appealing explanation for why $C$ should not be considered a cause of $E$ in this case: causes are difference makers, i.e., the value of $C$ should make a difference as to whether or not it causes $E$. In case of dependence, $C$ trivially makes such a difference, as in that case $C$ determines whether or not $E$ occurs at all. Cases of overdetermination and the like show that making a difference can be more subtle. These observations motivated Sartorio (2005) to propose the following principle: ${ }^{8}$

Principle 4 (Asymmetry). If $C$ is a cause of $E$ w.r.t. $(M, \vec{u})$, then $\neg C$ is not a cause of E w.r.t. $\left(M_{d o(\neg C)}, \vec{u}\right)$.

As Asymmetry and Transitivity focus on entirely different properties of causation, it is no surprise that they conflict with each other:

Theorem 3. Dependence, Transitivity, and Asymmetry are mutually inconsistent.

Theorem 3 teaches us that accepting Asymmetry provides an explanation for the fact that there are violations of Transitivity. ${ }^{9}$ In fact, picking up our earlier discussion, Asymmetry together with Contributing helps to make sense of Condition 2, which we can rephrase informally as:

If $C$ causes $D$ and $D$ causes $E$ w.r.t. $(M, \vec{u})$, then the following holds:

Transitivity should be respected unless this would violate Asymmetry.

There are now enough elements on the table to construct a coherent genesis of causation that explains its limited transitivity.

\subsection{Putting it All Together}

We started our analysis by noting the strong connection between dependence and causation. Specifically, by Dependence and Contributing, we know that causation lies somewhere in between dependence and contributing. Further, in the

8. Weslake (in press) adopts a similar-but not identical—principle.

9. Sartorio 2005 also uses Switches to argue that violations of Transitivity are due to Asymmetry. 
overwhelming majority of cases, all three of these concepts behave as a transitive relation. So as a first approximation, we assume causation to be some relation, say $\operatorname{Trans}(X, Y)$, which satisfies the following condition:

Condition 3. 1. $\operatorname{Trans}(X, Y)$ is transitive.

2. If Trans $(C, E)$ then $C$ contributes to $E$ w.r.t. $(M, \vec{u})$.

3. If $E$ depends on $C$ then Trans $(C, E)$ w.r.t. $(M, \vec{u})$.

The following generalisation of Theorem 1 offers a useful connection between dependence and such a $\operatorname{Trans}(X, Y)$ relation.

Theorem 4. If Trans $(X, Y)$ satisfies Condition 3 then:

$E$ depends on $C$ w.r.t. $(M, \vec{u})$ iff $\operatorname{Trans}(C, E)$ w.r.t. $(M, \vec{u})$ and $\operatorname{Trans}(\neg C, \neg E)$ w.r.t. $\left(M_{d o(\neg C)}, \vec{u}\right)$.

The following is a direct consequence of this theorem, which in analogy with Asymmetry we may call Anti-Symmetry.

Corollary 1 (Anti-Symmetry). If $E$ depends on $C$ w.r.t. $(M, \vec{u})$, then $\neg E$ depends on $\neg C$ w.r.t. $\left(M_{\text {do }(\neg C)}, \vec{u}\right)$.

Informally, this result tells us that dependence is built up out of any relation $\operatorname{Trans}(X, Y)$ that satisfies Condition 3 , in conjunction with the constraint that it should be Anti-Symmetrical.

Since for causation we only require Asymmetry, the solution is straightforward: causation is built up out of some relation $\operatorname{Trans}(X, Y)$ that satisfies Condition 3, in conjunction with Asymmetry. Putting all of this together, we get the following tentative characterisation of a good definition of causation:

Condition 4. There exists a relation Trans $(X, Y)$ such that each of the following holds:

1. $\operatorname{Trans}(X, Y)$ is transitive.

2. $C$ causes $E$ w.r.t. $(M, \vec{u})$ iff $\operatorname{Trans}(C, E)$ w.r.t. $(M, \vec{u})$ and $\neg \operatorname{Trans}(\neg C, E)$ w.r.t. $\left(M_{d o(\neg C)}, \vec{u}\right)$.

3. If Trans $(C, E)$ then $C$ contributes to $E$ w.r.t. $(M, \vec{u})$.

4. If E depends on $C$ then Trans $(C, E)$ w.r.t. $(M, \vec{u})$.

Any definition of causation satisfying the first and second part of Condition 4 is a compromise between Transitivity and Asymmetry: Transitivity is sacrificed only to the extent that is required to satisfy Asymmetry. Add to this the other two constraints, and we get a definition that has all the properties we have argued for. 
Theorem 5. Any definition of causation satisfying Condition 4 satisfies Dependence, Asymmetry, and Contributing, and Conditions 1 and 2

Since the weakest possible choice for Trans is to take contributing to, we state here the most straightforward definition of causation which meets all the demands of Condition 4.

Definition 6. Given $(M, \vec{u}) \mid=C \wedge E$, we define $C$ to be an actual cause of $E$ w.r.t. $(M, \vec{u})$ if $C$ contributes to $E$ w.r.t. $(M, \vec{u})$ and $\neg C$ does not contribute to $E$ w.r.t. $\left(M_{d o(\neg C)}, \vec{u}\right)$.

This definition gives the desired result for all of the examples discussed. We leave the details to the reader. (See the Appendix for details regarding Early Preemption.) However we consider Definition 6 too simple to be an adequate definition of causation in general. In this paper we focussed solely on issues arising from Transitivity and Asymmetry, but we have completely ignored issues related to the temporal properties of causation. More specifically, said definition is unable to deal with examples exhibiting Late Preemption, where an effect is overdetermined by two (or more) processes but only one of them deserves to be called a cause. In order to deal with those cases as well, and thus arrive at a definition of causation that is suited in general, the Trans relation should take into account temporal information as well. This is what we do in Beckers and Vennekens (in press), where we refine Definition 6 by developing the transitive notion of Production as a form of contributing that excludes preempted events. Specifically, we generalise the notion of production as introduced by Hall (2004), who uses it to highlight features of causation that are not found in dependence.

\section{Conclusion}

Starting from the observation that despite the intuitive appeal of the transitivity of causation there are many convincing counterexamples to accepting it, we have constructed an analysis in order to explain the precise relation between causation and transitivity. By pointing out the connection between violations of the transitivity of dependence, and violations of transitivity in general, we arrived at a characterisation of the transitivity of dependence that suggested a suitable sufficient condition for the transitivity of causation. Adding to this the principle of asymmetry resulted in a detailed genesis of causation, that narrows down the search to a proper definition of causation considerably. Finally, we have suggested a definition which meets all the requirements discussed, but remains incomplete until it is complemented with temporal properties that can handle Late Preemption. 


\section{Appendix}

\subsection{Generalising to Multi-valued Variables}

As mentioned in Section 2, throughout this work we have limited our discussion to models that only include Boolean variables. We now explain how we can generalise our results to also include structural models that contain multi-valued variables.

We defined a structural equation as taking on the form $V_{i}:=\phi$, where $\phi$ is a propositional formula over $\vec{V} \cup \vec{U}$, and we used $V_{i}$, respectively $\neg V_{i}$, as a shorthand for $V_{i}=$ true, respectively $V_{i}=$ false. In this more general setting, a structural equation takes the form $V_{i}:=F_{V_{i}}(\vec{W})$, where $F_{V_{i}}$ is a function from a set of variables $\vec{W} \subseteq(\vec{V} \cup \vec{U})$ to the domain of $V_{i}$. (The domains of each variable may vary, but they are all assumed finite.) As before, we only consider models $M$ such that the equations are acyclic.

Since the values of variables are no longer limited to true and false, the literals $C$ and $E$ that were used througout have to be written out explicitly as $C=c$ and $E=e$. Further, a negated atom like $\neg C$, i.e., $\neg(C=c)$, should be replaced with a formula $C=c^{\prime}$, and the condition that $c^{\prime} \neq c$.

For example, counterfactual dependence is now defined as:

Definition 7. Given a causal setting $(M, \vec{u})$ such that $(M, \vec{u}) \mid=C=c \wedge E=e, E=e$ is counterfactually dependent on $C=c$ if $\exists c^{\prime} \neq c, e^{\prime} \neq e:\left(M_{d o\left(C=c^{\prime}\right)}, \vec{u}\right) \models E=e^{\prime}$.

Except for explicitly writing out the literals, Condition 1 remains unchanged.

The generalisation of the definition of sufficiency is straightforward:

Definition 8. We define that a consistent set of literals $\vec{L}=\vec{l}$ is sufficient for a literal $L_{i}=l_{i}$ w.r.t. $M$ if $\vec{L}=\vec{l} \Rightarrow F_{L_{i}}\left(\vec{W}_{i}\right)=l_{i}$.

From there onwards, all definitions and theorems can be generalised in the same manner. Proofs of all theorems remain the same. Asymmetry, for example, now becomes:

Principle 4 (Asymmetry). If $C=c$ is a cause of $E=e$ w.r.t. $(M, \vec{u})$, then there exists a value $c^{\prime} \neq c$ so that $C=c^{\prime}$ is not a cause of $E=e^{\prime}$ w.r.t. $\left(M_{d o(}\left(C=c^{\prime}\right), \vec{u}\right)$.

Finally, the generalised version of our definition of actual causation becomes: Definition 9. Given $(M, \vec{u}) \mid=C=c \wedge E=e$, we define $C=c$ to be an actual cause of $E=e$ w.r.t. $(M, \vec{u})$ if $C=c$ contributes to $E=e$ w.r.t. $(M, \vec{u})$ and there exists a value $c^{\prime} \neq c$ so that $C=c^{\prime}$ does not contribute to $E=e$ w.r.t. $\left(M_{d o\left(C=c^{\prime}\right)}, \vec{u}\right)$.

\subsection{Actual Causation in Non-Deterministic Models}

As we mentioned in Section 4.1 when discussing Early Preemption, we accept a limited form of uncertainty regarding the actual story in judgments of actual cau- 
sation. We now make this formally precise, by specifying the level of information that is required for the notions of "actual cause" and "possible actual cause" to be used interchangeably.

Definition 10. We define that a partial causal setting $\left(M, \overrightarrow{u^{\prime}}\right)$ is actually complete if for any choice of $C$ and $E$, one of the two following conditions holds:

- $C$ certainly contributes to E w.r.t. $\left(M, \overrightarrow{u^{\prime}}\right)$.

- $C$ certainly does not contribute to E w.r.t. $\left(M, \vec{u}^{\prime}\right)$.

Given our definition of causation (Definition 6), an actually complete setting is one where all that is missing to be certain of the actual causes is information regarding the contributors in the counterfactual stories. This is precisely the situation that we encounter in our non-deterministic model for Backup from Section 4.1. the context tells us that Trainee certainly contributes to Victim, but in the counterfactual story all we can say is that Supervisor possibly contributes to Victim.

This gives rise to the following generalisation of actual causation to actually complete settings:

Definition 11. Given an actually complete causal setting $\left(M, \overrightarrow{u^{\prime}}\right)$ such that certainly $\left(M, \overrightarrow{u^{\prime}}\right)=C \wedge E$, we define $C$ to be an actual cause of $E$ w.r.t. $\left(M, \overrightarrow{u^{\prime}}\right)$ if $C$ certainly contributes to $E$ w.r.t. $\left(M, \overrightarrow{u^{\prime}}\right)$ and $\neg C$ possibly does not contribute to $E$ w.r.t. $\left(M_{d o(\neg C)}, \vec{u}^{\prime}\right)$.

Applying this definition to the non-deterministic model for Backup gives the desired result that Trainee is an actual cause of Victim.

The most general version of our definition of actual causation is given by the straightforward combination of Definitions 9 and 11 .

Definition 12 (Actual Causation). Given an actually complete causal setting $\left(M, \overrightarrow{u^{\prime}}\right)$ such that certainly $\left(M, \overrightarrow{u^{\prime}}\right) \models C=c \wedge E=e$, we define $C=c$ to be an actual cause of $E=e$ w.r.t. $\left(M, \overrightarrow{u^{\prime}}\right)$ if $C=c$ certainly contributes to $E=e$ w.r.t. $\left(M, \overrightarrow{u^{\prime}}\right)$ and there exist a value $c^{\prime} \neq c$ so that $C=c^{\prime}$ possibly does not contribute to $E=e$ w.r.t. $\left(M_{d o\left(C=c^{\prime}\right)}, \overrightarrow{u^{\prime}}\right)$.

It is clear that if the actually complete causal setting is non-partial (i.e., the context stipulates the value of each exogenous variable) and all variables are Boolean, then Definition 12 reduces to Definition 6.

8.3. Proofs of Theorems

Theorem 1. E depends on $C$ w.r.t. $(M, \vec{u})$ iff $C$ contributes to $E$ w.r.t. $(M, \vec{u})$ and $\neg C$ contributes to $\neg E$ w.r.t. $\left(M_{d o}(\neg C), \vec{u}\right)$.

Proof. The implication from right to left is trivial, so we only prove the implication from left to right. So assume $E$ depends on $C$ w.r.t. $(M, \vec{u})$, or in other words, $(M, \vec{u}) \models C \wedge E$ and $\left(M_{d o(\neg C)}, \vec{u}\right) \mid \neg E$. 
We first prove that $C$ contributes to $E$ w.r.t. $(M, \vec{u})$.

Take $L^{1}$ to be minimally sufficient for $E$, i.e., $L^{1}$ is sufficient for $E$, and for any $L_{i} \in L^{1}, L^{1} \backslash\left\{L_{i}\right\}$ is not sufficient for $E$. (Such a set can be constructed by removing elements from a sufficient set $\vec{L}$ one by one.) By construction, all endogenous literals in $L^{1}$ are direct actual contributors to $E$.

By $L_{(M, \vec{u})}$ we denote all literals $L_{i}$ such that $(M, \vec{u}) \models L_{i}$.

Since $\vec{U}=\vec{u} \subset L_{\left(M_{d o(\neg C)}, \vec{u}\right)}$, it follows that if $L^{1} \backslash \vec{U}=\vec{u} \subseteq L_{\left(M_{d o(\neg C)}, \vec{u}\right)}$, then $E \in$ $L_{\left(M_{d o(\neg C)}, \vec{u}\right)}$, i.e., $\left(M_{d o(\neg C)}, \vec{u}\right) \models E$. Therefore there exists at least one endogenous literal $D \in L^{1}$ such that $D \notin L_{\left(M_{d o(\neg C)}, \vec{u}\right)}$. By the previous paragraph, $D$ is a direct actual contributor to $E$.

If $D=C$, then we are finished with this part of the proof. So assume $D \neq C$. We can apply the exact same reasoning as we did for $E$, to find a direct actual contributor $F$ to $D$ such that $F \notin L_{\left(M_{d o(\neg C)}, \vec{u}\right)}$. Since contributing is transitive, $F$ contributes to $E$ as well. Given that there are only a finite number of endogenous literals, and that $M$ is assumed to be acyclical, continuing this reasoning will eventually end up with finding $C$ as an actual contributing cause of $E$. Therefore we conclude that $C$ contributes to $E$ w.r.t. $(M, \vec{u})$.

We can apply the exact same reasoning to prove that also $\neg C$ contributes to $\neg E$ w.r.t. $\left(M_{d o(\neg C)}, \vec{u}\right)$, which concludes the proof.

Theorem 2. If $E$ depends on $D$ and $D$ depends on $C$ w.r.t. $(M, \vec{u})$, then the following statements are all equivalent:

1. E depends on $C$ w.r.t. $(M, \vec{u})$.

2. $\neg$ E depends on $\neg C$ w.r.t. $\left(M_{\text {do }(\neg C)}, \vec{u}\right)$.

3. $\neg C$ contributes to $\neg E$ w.r.t. $\left(M_{d o(\neg C)}, \vec{u}\right)$.

4. $\neg C$ does not contribute to E w.r.t. $\left(M_{d o(\neg C)}, \vec{u}\right)$.

Proof. Assume $E$ depends on $D$ and $D$ depends on $C$ w.r.t. $(M, \vec{u})$. First, note that by Theorem $I$ this implies that $C$ contributes to $D$ and $D$ contributes to $E$ w.r.t. $(M, \vec{u})$. Since contributing is transitive by construction, this implies that $C$ contributes to $E$ w.r.t. $(M, \vec{u})$.

We start with assuming that $E$ depends on $C$ w.r.t. $(M, \vec{u})$. It follows directly from the definitions that this is equivalent to $\neg E$ depends on $\neg C$ w.r.t. $\left.\left(M_{d o} \neg C\right), \vec{u}\right)$.

Now assume we know that $\neg E$ depends on $\neg C$ w.r.t. $\left(M_{d o(\neg C)}, \vec{u}\right)$. Given that we already know that $C$ contributes to $E$ w.r.t. $(M, \vec{u})$, by Theorem 1 we see that this is equivalent to $\neg C$ contributes to $\neg E$ w.r.t. $\left(M_{d o}(\neg C), \vec{u}\right)$.

Lastly, assume that $\neg C$ contributes to $\neg E$ w.r.t. $\left(M_{d o(\neg C)}, \vec{u}\right)$. It follows directly that $\neg C$ does not contribute to $E$ w.r.t. $\left(M_{d o(\neg C)}, \vec{u}\right)$. Remains the reverse implication. It suffices to show that if $\neg C$ does not contribute to $E$ w.r.t. $\left(M_{d o(\neg C)}, \vec{u}\right)$, then $\left(M_{d o(\neg C)}, \vec{u}\right) \mid \neg E$. 
We proceed by a reductio: assume that $\neg C$ does not contribute to $E$ w.r.t. $\left(M_{d o(\neg C)}, \vec{u}\right)$ and $\left(M_{d o(\neg C)}, \vec{u}\right) \mid=E$.

$D$ depends on $C$ w.r.t. $(M, \vec{u})$, and thus $\left(M_{d o(\neg C)}, \vec{u}\right) \models \neg D$. Together with the fact that $\left(M_{d o(\neg C)}, \vec{u}\right) \models E$, this implies that $\left(M_{d o(\neg C, \neg D)}, \vec{u}\right) \models E$. Also, since $E$ depends on $D$ w.r.t. $(M, \vec{u})$, we have $\left(M_{d o(\neg D)}, \vec{u}\right) \mid=\neg E$. Therefore $\neg E$ depends on $C$ w.r.t. $\left(M_{d o(\neg D)}, \vec{u}\right)$. By Theorem 1 , this implies that $\neg C$ contributes to $E$ w.r.t. $\left(M_{d o(\neg C, \neg D)}, \vec{u}\right)$, and thus also $\neg C$ contributes to $E$ w.r.t. $\left(M_{d o(\neg C)}, \vec{u}\right)$, which concludes the proof.

Theorem 3. Dependence, Transitivity, and Asymmetry are mutually inconsistent.

Proof. We have a look again at the Switch example. In the story such that Switch holds, by Dependence Switch is a cause of $L T$ and $L T$ is a cause of Dest. By Transitivity, this makes Switch a cause of Dest. But if we look at the story do ( $\neg$ Switch), then we can apply the same reasoning to get that $\neg$ Switch is a cause of Dest. This is in violation of Asymmetry.

Theorem 4. If Trans $(X, Y)$ satisfies Condition 3 then:

$E$ depends on $C$ w.r.t. $(M, \vec{u})$ iff $\operatorname{Trans}(C, E)$ w.r.t. $(M, \vec{u})$ and $\operatorname{Trans}(\neg C, \neg E)$ w.r.t. $\left(M_{d o(\neg C)}, \vec{u}\right)$.

Proof. We start with the implication from left to right. So assume $E$ depends on $C$ w.r.t. $(M, \vec{u})$, which is equivalent to $\neg E$ depends on $\neg C$ w.r.t. $\left(M_{d o(\neg C)}, \vec{u}\right)$. Hence by applying 3 to both statements, we get the desired result.

Remains the implication from right to left. So assume $\operatorname{Trans}(C, E)$ w.r.t. $(M, \vec{u})$ and Trans $(\neg C, \neg E)$ w.r.t. $\left(M_{d o(\neg C)}, \vec{u}\right)$. By 2, this implies that $C$ contributes to $E$ w.r.t. $(M, \vec{u})$ and $\neg C$ contributes to $\neg E$ w.r.t. $\left(M_{d o(\neg C)}, \vec{u}\right)$. Applying Theorem $\mathbb{I}$ gives the result.

Theorem 5. Any definition of causation satisfying Condition 4 satisfies Dependence, Asymmetry, and Contributing, and Conditions 1 and 2

Proof. Dependence: Assume $E$ depends on $C$ w.r.t. $(M, \vec{u})$. By 2, we need to show that $\operatorname{Trans}(C, E)$ w.r.t. $(M, \vec{u})$ and $\neg \operatorname{Trans}(\neg C, E)$ w.r.t. $\left(M_{d o(\neg C)}, \vec{u}\right)$. The former is a direct consequence of 4 , so remains the latter.

Since $E$ does not hold in $\left(M_{d o(\neg C)}, \vec{u}\right)$, we get that $\neg C$ does not contribute to $E$ w.r.t. $\left(M_{d o(\neg C)}, \vec{u}\right)$. By 3 , this implies that $\neg \operatorname{Trans}(\neg C, E)$ w.r.t. $\left(M_{d o(\neg C)}, \vec{u}\right)$.

Asymmetry and Contributing follow immediately from 2 and 3.

Condition 1: Assume $E$ depends on $D$ and $D$ depends on $C$ w.r.t. $(M, \vec{u})$. By 1 and 4 this implies that $\operatorname{Trans}(C, E)$ w.r.t. $(M, \vec{u})$. The implication from right to left in the equivalence from Condition 1 follows from Dependence. So we need to prove that $\neg \operatorname{Trans}(\neg C, E)$ w.r.t. $\left(M_{d o(\neg C)}, \vec{u}\right)$ implies that $E$ depends on $C$ w.r.t. $(M, \vec{u})$. 
We proceed by a reductio: assume that $\neg \operatorname{Trans}(\neg C, E)$ w.r.t. $\left(M_{d o(\neg C)}, \vec{u}\right)$ and $\left(M_{d o(\neg C)}, \vec{u}\right) \mid=E$.

$D$ depends on $C$ w.r.t. $(M, \vec{u})$, and thus $\left(M_{d o(\neg C)}, \vec{u}\right) \mid=\neg D$. Together with the fact that $\left(M_{d o(\neg C)}, \vec{u}\right) \models E$, this implies that $\left(M_{d o(\neg C, \neg D)}, \vec{u}\right) \models E$. Also, since $E$ depends on $D$ w.r.t. $(M, \vec{u})$, we have $\left(M_{d o(\neg D)}, \vec{u}\right) \models \neg E$. Therefore $\neg E$ depends on $C$ w.r.t. $\left(M_{d o(\neg D)}, \vec{u}\right)$. By Theorem 4, this implies that $\operatorname{Trans}(\neg C, E)$ w.r.t. $\left(M_{d o(\neg C, \neg D)}, \vec{u}\right)$, and thus also $\operatorname{Trans}(\neg C, \vec{E})$ w.r.t. $\left(M_{d o(\neg C)}, \vec{u}\right)$, which concludes the proof.

Condition 2: Assume $C$ causes $D$ and $D$ causes $E$ w.r.t. $(M, \vec{u})$, and $\neg C$ does not contribute to $E$ w.r.t. $\left(M_{d o(\neg C)}, \vec{u}\right)$. By 1 , we get that $\operatorname{Trans}(C, E)$ w.r.t. $(M, \vec{u})$. By 3, we also get that $\neg \operatorname{Trans}(\neg C, E)$ w.r.t. $\left(M_{d o(\neg C)}, \vec{u}\right)$, and thus $C$ causes $E$ w.r.t. $(M, \vec{u})$.

\section{Acknowledgements}

The authors would like to thank two anonymous reviewers and the editor for many helpful comments on an earlier version of this text. Sander Beckers was funded by the Flemish Agency for Innovation by Science and Technology (IWT).

\section{References}

Beckers, Sander and Joost Vennekens (in press). A Principled Approach to Defining Actual Causation. Synthese.

Hall, Ned (2000). Causation and the Price of Transitivity. Journal of Philosophy, 97(4), 198-222.

Hall, Ned (2004). Two Concepts of Causation. In John Collins, Ned Hall, and Laurie Ann Paul (Eds.), Causation and Counterfactuals (225-276). The MIT Press.

Hall, Ned (2007). Structural Equations and Causation. Philosophical Studies, 132(1), 109-136.

Halpern, Joseph (2016a). Actual Causality. MIT Press.

Halpern, Joseph (2016b). Sufficient Conditions for Causality to Be Transitive. Philosophy of Science, 83(2), 213-226.

Halpern, Joseph and Christopher Hitchcock (2010). Actual Causation and the Art of Modeling. In Rina Dechter, Hector Geffner, and Joseph Halpern (Eds.), Heuristics, Probability and Causality: A Tribute to Judea Pearl (383-406). College Publications.

Halpern, Joseph and Judea Pearl (2005). Causes and Explanations: A StructuralModel Approach. Part I: Causes. The British Journal for the Philosophy of Science, 56(4), 843-87.

Hitchcock, Christopher (2001). The Intransitivity of Causation Revealed in Equa- 
tions and Graphs. Journal of Philosophy, 98(6), 273-299.

Hitchcock, Christopher (2009). Structural Equations and Causation: Six Counterexamples. Philosophical Studies, 144(3), 391-401.

Lewis, David (1973). Causation. Journal of Philosophy, 70(17), 113-126.

Lewis, David (1986). Causation. In Philosophical Papers Volume II (159-213). Oxford University Press.

Lewis, David (2000). Causation as Influence. Journal of Philosophy, 97(4), 182-197. McDermott, Michael (1995). Redundant Causation. The British Journal for the Philosophy of Science, 46(4), 523-544.

Menzies, Peter (2004). Causal Models, Token Causation, and Processes. Philosophy of Science, 71(5), 820-832.

Paul, Laurie Ann and Ned Hall (2013). Causation: A User's Guide. Oxford University Press.

Pearl, Judea (200o). Causality: Models, Reasoning, and Inference. Cambridge University Press.

Sartorio, Carolina (2005). Causes As Difference-Makers. Philosophical Studies, $123(1), 71-96$.

Weslake, Brad (in press). A Partial Theory of Actual Causation. The British Journal for the Philosophy of Science.

Woodward, James (2003). Making Things Happen: A Theory of Causal Explanation. Oxford University Press. 